\title{
MOYAMOYA DISEASE WITH REVIEW OF LITERATURE
}

Mir Sumsam Ali Khurram¹, S. Pratap Rao², Ahmedullah Khan ${ }^{3}$

\section{HOW TO CITE THIS ARTICLE:}

Mir Sumsam Ali Khurram, S Pratap Rao, Ahmedullah Khan. "Moyamoya disease with review of literature". Journal of Evolution of Medical and Dental Sciences 2013; Vol2, Issue 48, December 02; Page: 9419-9422.

ABSTRACT: Moyamoya disease is an angiogenic disorder and a rare cause of stroke, it is a progress narrowing of cerebral arteries at the base of the brain involving the intracerebral portion of the internal carotid arteries, where it leads to development of collateral arteries, causing a "cigarette smoke" aspect. We report a case of 9 years old male child who was admitted in our institution with the complaint of vomiting, weakness over the right upper and lower limb, facial palsy and speech disturbances. On C.T, angiography the child was diagnosed to have moyamoya disease.

KEYWORD: Moyamoya disease, right hemiplegia, facial palsy, MRI, C.T. Angiography.

INTRODUCTION: Moyamoya (A Japanese term) meaning hazy things such as puff of smoke and was first described by Takeuchi and Shimizu in $1957\{2,4,5\}$ the over all incidence is higher (0.35 per $1,00,00)$ Takeuchi was distinguished two forms of this disease as primary moyamoya disease and secondary moyamoya disease. Moyamoya disease is a rare cerebrovascular disease of known aetiology $\{1\}$ we reported a case of 9 years old male child who presented with right hemiplegia, Facial Palsy and disturbed speech at Owaisi hospital and research centre attached to Deccan College of Medical Sciences Hyderabad, A.P. India.

CASE REPORT: A 9 years old male child was admitted in our institution with a complaints of loose motion and vomiting, the child was initially treated as acute gastroenteritis. Three days later the child developed sudden onset of weakness over right upper \& right lower limb, and deviation of the mouth to the left side. Over a period of 1 week, the child was developed altered Sensorium and slurred speech. There was history of convulsion at the age of 4 years for which, the child was treated as a case of febrile convulsion. There was no history of fever, head injury and delayed milestone. On neurological examination right facial palsy was detected, gait was hemiplegic, tone was decreased over right side.

Power was 2/5 over right upper and right lower limb, deep tendon reflexes was exaggerated, and planter was extensor over right side. No signs of meningeal irritation. CSF examination was normal.

Laboratory investigation showed: CBC! HB 11.6 g per d/l, MCV-77, WBC: 12,000/3mm, platelet: 4,50,000 L / mm.

ABG PH; 7.42, Pco2 34, Po2 72 HC03:2, Oxygen Saturation was 99\%on RA.

Calcium: $9.4 \mathrm{mg} / \mathrm{d} / \mathrm{l}$, creatinine $0.4 \mathrm{mg} / \mathrm{d} / \mathrm{l}$.

Chest X-Ray revealed left upper lobe hazy infiltration.

C.T. Scan of brain showed large infarct involving anterior cerebral artery and middle cerebral artery territory. (Fig - 1).

CT Angiography of brain showed evidence of distal internal carotid arteries and proximal portion of anterior cerebral artery and middle cerebral artery on right side with associated leptomeningeal collateral. (Fig - 2). Thus CT angiography suggestive of moyamoya disease. 
The patient was treated with supportive measure correction of fluids and electrolytes, vitamins, physiotherapy, vasodilators and anticoagulants was prescribed. His general condition was improved especially weakness of his right upper \& right lower limb significantly decreased. The facial paralysis of the right side resolved. No convulsion was noticed during 12 days of hospitalization. Follow-up evaluation showed nearly resolution of his right sided weakness. Gait was returned to normal condition and the child was able to move his right hand and leg freely.

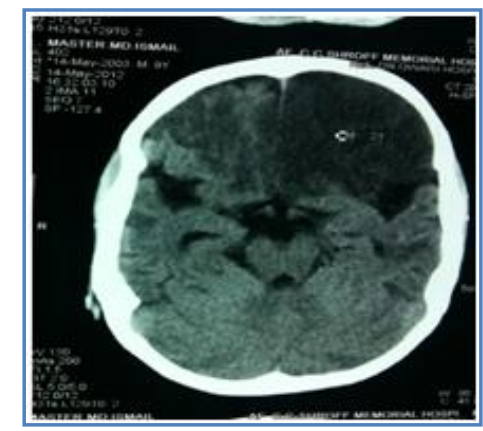

Fig. 1: CT Scan of the head Showing large infarct involving anterior cerebral artery and middle cerebral artery territory.

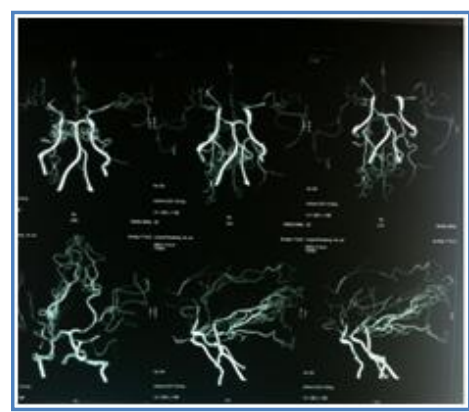

Fig.2: CT angiography of the brain showing evidence of occlusion of distal internal carotid arteries and proximal portions of anterior cerebral artery and middle cerebral artery on the right side with associated leptomeningeal collaterals (ivy sign).

DISCUSSION: Moyamoya disease is a progressive occlusive disease of the cerebral vasculature with particular involvement of the circle of the willis.

Moyamoya disease is categorised as either ischemic and haemorrhagic type or true mixed type and the etiology of this disease is unknown $\{5\}$.

There are 2 types of moyamoya disease as primary \& secondary.

Primary moyamoya disease is common among Japanese patients. The incidence is 0.1 in $1,00,000$, per year. In pediatric age group, girls are more frequently affected, with the peak age of onset before 5 years of age $\{1,3,5 \& 6\}$.

In children, the most common presentation includes recurrent episodes of cerebral ischemia manifesting clinically as facial deficits, paresthesia, and seizures $\{1\}$. The initial symptoms include motor disturbances, an alternating hemiparesis, transient ischemic attack, speech disturbances and seizures. 
Mental deterioration seen in approximately one third of the child.

Angiography or MRI scan shows several stages of primary moyamoya disease. Secondary moyamoya disease is caused by a variety of underlying disorders such as sickle anemia, neurofibromatosis-I, downs syndrome, congenital heart defects, renal artery stenosis and thyroiditis have been found to be associated with moyamoya disease $\{5\}$, but more than half of the children seen with disease have no cause for their moyamoya syndrome.

In our case the diagnosis was mainly based on clinical features of stroke (Hemiplegia), facial palsy, slurred speech and imaging finding.

Moyamoya disease should be considered in any patient with acute symptoms of stroke, and this disease shows various neurological signs.

The neurological manifestation could be varied ranging from transient ischemic attach (TIA) to extensive bilateral strokes, involuntary movements, seizure and speech disturbances. Some children may have mental retardation due to long term ischemia or repeated infarct.

Cerebral angiography is used to confirm the diagnosis and typically reveals the narrowing and occlusion of proximal cerebral vessels and extensive collateral flow through the perforating vessels showing the classical puff of smoke appearance $\{7\}$.

Management of Moyamoya disease is mainly symptomatic, patients effecting strokes should be recommended aspirin, vasodilators, anticoagulant, and surgical treatment has been successful, especially among the children.

Revascularization procedures are currently performed in increase the perfusion to hypoxic brain.

Revascularization produces direct, indirect or combined anastomotic revascularization.

Direct revascularization includes anastomosis of the superficial temporal artery or occipital artery to the middle cerebral artery.

Indirect revascularization surgery for moyamoya disease result in the development of collaterals from the external carotid arterial system into the middle cerebral artery $\{14$ to 16$\}$

\section{REFERENCES:}

1. Fujii K, IkezakiK, Irikru K, Miyasaka Y, Fukui M. The efficacy of bypass surgery for the patient with hemorrhagic moyamoya disease. Clin Neurol Neurosurg 1997 : 99 (Suppl): 194-5.

2. Fukui M. Current state of study on moyamoya disease in Japan surg. Neural. $1997 ; 47: 138$ 43.

3. Kim SK Seol HJ, Cho BK, Hwang YS, Lee DS, Wang KC, Moyamoya disease among young patients, its aggressive clinical course and the role of the active surgical treatment. Neurosurgery 2004 ; 54; 840-60.

4. Kobayashi E, Sacki N, Oishi H, Hirais, Yamaura A, Long term natural history of haemorrhagic moyamoya disease in 42 patients. J. Neuro surg. 2000; 93; 976-80.

5. Y. Natori, K. Kjezaki, T. Matsushima and M. Fukui "Angiography moyamoya its definition, classification and therapy, Clinical, neurology and neurosurgery, Vol, 99 No.2 PPS 168-S172, 1997.

6. I, Lutter man, M. Scott, R. Naes and T. Geva "Moyamoya syndrome associated with congenital heart disease" pediatrics, vol 101, No. 1, PP 57 - 601998 View at publisher. 
7. E. R. Smith R.M. Scott, moyamoya syndrome associated with congenital heart disease, skull base, Vol. 15 No 1PP.

8. D.H. Han, O.K. Kwon, B.J. Bark Jang Byun etal, "A cooperative study; clinical characteristics of 334 Korean patients with moyamoya disease treated at neurosurgical institution (976-1994), Acta neurochirugica. Vol. 142, No. 11, PP. 1263 - 12742000.

9. Andrew R. Gregory W. Garyk. Moyamoyan Syndrome in children with Alagile syndrome pediatrics 1999, 103; 505-508.

10. Adams RD. Cerebrovascular disease. Principles of neurology. New York. 1993 P. 669-748.

11. Bruno A. Adams HP, Biller J, Rezai K, Cornell S. Cerebral infraction due to moyamoya disease in young adult. Stroke 1988, 19: 826-833.

12. Tomura N, Ingami A, Hingano S. Moyamoya Syndroe in young children Shinkei 1988 Oct; 40: 905-912.

13. Matsushima, Inaba Y. Moyamoya disease in children and its surgical treatment child brain 1984;11: 155-170.

14. Suzuki, J. Kodama N. Moyamoya disease; the disorder and surgical treatment moya clinical procedure 1983; 69: 749-457.

\section{AUTHORS:}

1. Mir Sumsam Ali Khurram

2. S. Pratap Rao

3. Ahmedullah Khan

\section{PARTICULARS OF CONTRIBUTORS:}

1. Associate Professor, Department of Pediatrics, Deccan College of Medical Sciences.

2. Professor, Department of Pediatrics, Deccan College of Medical Sciences.

3. Post Graduate, Department of Pediatrics, Deccan College of Medical Sciences.

\section{NAME ADRRESS EMAIL ID OF THE} CORRESPONDING AUTHOR:

Dr. Mir Sumsam Ali Khurram, H.No. 23-1-1027, Inside Moghalpura, Kaman Hyderabad, Andhra Pradesh, India. Email - dr.sakhurram@gmail.com

Date of Submission: 14/11/2013.

Date of Peer Review: 15/11/2013.

Date of Acceptance: 21/11/2013.

Date of Publishing: 29/11/2013 